\title{
Clinical Intuition in Family Medicine: More Than First Impressions
}

\author{
Amanda Woolley, BA \\ Olga Kostopoulou, MSc, PbD
}

Department of Primary Care and Public Health Sciences, School of Medicine, King's College London, London, United Kingdom

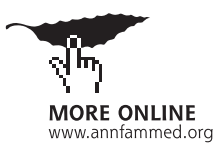

Conflicts of interest: authors report none.

\section{CORRESPONDING AUTHOR}

Olga Kostopoulou, MSc, PhD

Department of Primary Care and Public

Health Sciences

School of Medicine

King's College London

Capital House, 42 Weston St

London SE1 3QD, UK

olga.kostopoulou@kcl.ac.uk

\begin{abstract}
PURPOSE The clinical literature advises physicians not to trust their intuition. Studies of clinical intuition, however, equate it to early impressions, the first thing that comes to the physician's mind. This study aimed to investigate the validity of this perspective by examining real cases of intuition in family medicine.

METHOD Eighteen family physicians were interviewed about patient cases in which they believed that they had experienced an intuition. Cases were included if (1) participants were unaware of the basis of their judgment, or (2) participants talked about the basis of their judgment but believed that it was irrational or unsubstantiated. During the interview, case descriptions were systematically probed following the Critical Decision Method. Transcripts were coded for judgments, informational cues, expectancies, goals, and actions and were reordered into chronological accounts of the decision process. The 2 authors independently categorized cases into 3 emerging decision process types.
\end{abstract}

RESULTS Participants reported 31 cases, 24 of which met inclusion criteria. Three types of decision process emerged: gut feelings, recognitions, and insights $(\kappa=0.78)$. In all cases, participants thought that their intuitive judgment was in conflict with a more rational explanation or what other colleagues would do.

CONCLUSION Automatic, nonanalytical processes in clinical judgment extend beyond first impressions. Rather than admonishing clinicians not to trust their intuition, it should be acknowledged that little is currently known about the different types of intuitive processes and what determines their success or failure. Research on the conditions for accurate clinical intuitions is needed.

Ann Fam Med 2013;11:60-66. doi:10.1370/afm.1433.

\section{INTRODUCTION}

$\mathrm{T}$ There is a recognized tension between clinical intuition being seen as the mark of an expert and as mere guesswork, unnecessary in the age of evidence-based medicine. ${ }^{1}$ Both perspectives agree that intuition eludes understanding, which contributes to the interest it generates. ${ }^{2,3}$ Intuition, ie, making judgments without any awareness of reasoning, however, has been the subject of robust scientific inquiry in psychology for at least 40 years. Intuitive decision making has been found in some cases to improve decisions ${ }^{4-6}$ and lead consistently to better performance than analytic deliberation. ${ }^{7-9}$ The clinical literature nevertheless warns doctors against intuition to avoid reasoning errors and cognitive biases. ${ }^{10-13}$ Much of this literature is opinion-based and attempts to apply psychological concepts to medical diagnosis without empirical testing.

A small number of empirical studies have attempted to compare diagnostic accuracy under conditions of analytical vs nonanalytical processing with mixed results. For example, residents in internal medicine who were instructed to apply an analytical approach diagnosed complex cases more accurately than those relying on first impressions. ${ }^{14}$ Studies of visual diagnosis using student participants produced opposite results: accuracy diagnosing dermatological slides_-similar to those seen in training — was 
higher among those instructed to give the first thing that came to mind than those using careful analysis. ${ }^{15}$ In another study, accuracy in interpreting electrocardiograms was found to improve when participants were prompted to use a combination of analytical and intuitive strategies. ${ }^{16}$

These studies equate nonanalytical responses with first impressions. An instruction to rely on first impressions encourages consideration of only the most easily recalled information, which is likely to be the most common disease or the most typical presentation. Thus, although claims about the deleterious effects of clinical intuition are based on these studies, ${ }^{10,12}$ their results can only generalize to the quick, associative memory processes that use the most easily accessible information and often occur at the start of the doctorpatient encounter. It is arguable to what extent first impressions are representative of clinical intuition. Clinicians tend to describe their intuitions as gut feelings, admit not knowing their basis, and consider them irrational. ${ }^{17}$ In contrast, first impressions can be considered perfectly rational and justifiable.

Intuition research in psychology has extended beyond first impressions. It has studied heuristic-based decision making in which heuristics are nonconscious, simple decision rules that use little information, thereby reducing cognitive effort, usually without harming performance. ${ }^{4,18,19}$ For example, in situations where the decision maker lacks the necessary knowledge to make an informed choice between alternatives, the alternative that has been encountered before is more likely to be selected on the basis of this recognition alone (the recognition heuristic). ${ }^{20}$ Dual process theories contrast intuitive or system 1 processes (fast, parallel, effortless, and emotion based) with analytical, system 2 processes (slow, serial, effortful, and rule based) ${ }^{21}$; researchers have recently focused on how the transition from intuitive to a more analytical mode of reasoning occurs. ${ }^{22,23}$ The surprising "deliberation without attention" effect has also been the focus of several recent studies. In situations where working memory capacity is too limited for the amount of information that needs to be processed, a short distraction from the task was sometimes shown to improve decision making when compared with thinking analytically for the same length of time. ${ }^{24}$ People have also been found to make accurate judgments about large and complex information sets based on implicit knowledge, ie, knowledge of which they are not consciously aware. ${ }^{5,25}$ This abundance of research reflects the plurality of views about intuitive processes and the circumstances that give rise to them in both experimental and naturalistic settings.

Our study aimed to examine intuition as experienced by physicians and to identify the cognitive processes active in medical decision making. This reevaluation of clinical intuition stands between extant literature on the usefulness of intuitive processes in medicine and the everyday experiences of practicing physicians. ${ }^{6}$

\section{METHODS}

Participants were family physicians practicing in London, United Kingdom. There were 2 criteria for recruitment: sex and experience. First, we aimed to recruit male and female family physicians in equal numbers because of sex differences found in the ability to inhibit an intuitive response. ${ }^{26}$ Second, guided by the 10 -year rule in the expertise literature, ${ }^{27,28}$ we aimed to recruit physicians with more than 10 years and with 10 or fewer years' experience in family medicine in equal numbers. Academic family physicians at King's College London were invited to participate and asked to forward the invitation to other practicing family physicians outside academia. Data collection ceased when the sex and experience criteria were fulfilled and theoretical saturation was reached, ie, no new types of decision processes were being discussed. Ethical approval was obtained from the North West London Research Ethics Committee 2 (10/H0720/50).

\section{Procedure}

A week before the scheduled interview, participants were sent a standard e-mail asking them to think of 2 occasions when they felt they knew the diagnosis or prognosis of a patient but did not know how they knew: one case for which their feeling was correct and one for which it was incorrect. We requested 2 cases to overcome an anticipated bias to recall only positive instances. Interviews were conducted at the participant's clinic or the university and took 1 hour.

At the start of the interview, participants gave a brief summary of each case that they had in mind. Based on this summary, the interviewer (A.W.) judged the case to meet inclusion criteria if (1) participants were unaware of the basis of their judgment, or (2) participants talked about the basis of their judgment but believed that it was irrational or unsubstantiated. Cases deemed unsuitable at this stage were not discussed further.

The Critical Decision Method was used to conduct the interviews. ${ }^{29}$ This semistructured interview method elicits the cues (pieces of information), expectancies, and goals associated with judgments made using expert intuition (Table 1). ${ }^{30}$ The Critical Decision Method focuses on systematically probing events that lead up to a decision and aims to uncover the implicit information that the decision maker used and the cognitive processes that were active at the time. Because case 


\begin{tabular}{|c|c|}
\hline Steps & Description \\
\hline Step 1 & $\begin{array}{l}\text { The participant defines the scope of the event, ie, whether it took place during a } \\
\text { single consultation or over multiple consultations. The scope is refined, if neces- } \\
\text { sary, by the interviewer to cover only the participant's involvement. For example, } \\
\text { details of patients' secondary care and outcome or related consultations with other } \\
\text { family physicians were noted but not probed }\end{array}$ \\
\hline Step 2 & $\begin{array}{l}\text { The interviewer repeats back the account to be updated or corrected until a shared } \\
\text { understanding of the episode is reached }\end{array}$ \\
\hline Step 3 & $\begin{array}{l}\text { A timeline of events is drawn on paper to aid accurate elicitation of decision infor- } \\
\text { mation, paying attention to when new information was received and when key } \\
\text { decisions were made }\end{array}$ \\
\hline Step 4 & $\begin{array}{l}\text { Using the timeline, the interviewer probes the account for more detail at points of } \\
\text { new information, judgments, decisions, and reasoning processes. In this way, the } \\
\text { implicit information and processes that were active during the episode are elicited }\end{array}$ \\
\hline Step 5 & $\begin{array}{l}\text { Hypothetical questions are asked to identify the implicit cues critical to intuitive } \\
\text { decisions, for example, "What if the blood results were normal? How would that } \\
\text { change your appraisal?" }\end{array}$ \\
\hline
\end{tabular}

each physician because of time constraints. In yet another interview, the audio recording failed, and no cases were recorded. As a result, 31 rather than 36 cases were obtained from the 18 interviewees. During subsequent analyses of the transcripts, the authors agreed that 7 more cases did not meet inclusion criteria, resulting in a total of 24 cases of intuition.

Three types of decision process emerged during iterative reading of the transcripts: gut feelings, recognitions, and insights. Case examples for each descriptions were based on memory, the Critical Decision Method was used to ensure more complete and internally consistent descriptions.

Interviews were audio-recorded and transcribed. All personal or identifying information regarding participants, patients, and colleagues was removed from the transcripts.

\section{Analysis}

Any unclear points in the transcripts were discussed with academic physicians at the authors' institution to ensure that the clinical content of the cases was well understood. The first author coded each transcript for judgment points and related cues, expectancies, goals, and actions (Table 2). Coded statements relating to a single judgment point were then arranged into chronological protocols, as advised when using the Critical Decision Method. ${ }^{29}$ Protocols were subsequently compared with each other in terms of the timing of the intuition, the cues available at that time, and the interpretation of subsequent information. The 2 authors independently categorized all the transcripts into 3 types of decision process that emerged during analysis. Subsequently, disagreements in case categorization were discussed and full agreement reached.

\section{RESULTS}

Participants were 18 family physicians in London, United Kingdom (9 female; mean age $=44$ years, $\mathrm{SD}=14$ years). Nine of the participants had been practicing family medicine for longer than 10 years (mean $=27$ years, SD $=10$ years; 5 female) and the other 9 had been practicing for 10 or fewer years (mean $=3$ years, $\mathrm{SD}=3$ years 4 female).

In 1 interview, only 1 case met inclusion criteria. In 2 other interviews, only 1 case was elicited from type are provided in the Supplemental Appendix, available at http://annfammed.org/content/11/1/60/ suppl/DC1. Good agreement was achieved in the allocation of cases to process types $(\kappa=0.78)$.

\section{Gut Feelings}

This case type was most frequently reported and constituted one-half of the cases included in the analysis. In gut feeling cases, the physician formed an initial interpretation based on the patient's reason for the encounter and/or the existing notes in the patient's chart. During further information gathering, an intuition experienced as a feeling cast doubt over the initial interpretation. In all gut feeling cases reported, the intuition signaled alarm, often in response to a single cue that "did not seem right" or an unexpected pattern of cues. In most cases, participants were aware of some basis to their judgment but thought that it was not evidence based or supported by guidelines. They were not always certain about what the nonfitting cues meant

\begin{tabular}{|c|c|}
\hline Criteria & Description \\
\hline Judgments & $\begin{array}{l}\text { The point of making a judgment, appraisal, current } \\
\text { hypotheses, or working diagnosis }\end{array}$ \\
\hline Cues & $\begin{array}{l}\text { All perceptual and verbal information available } \\
\text { explicitly and elicited implicit cues, including } \\
\text { symptoms, signs, and context. Information } \\
\text { learned after the episode, therefore not associ- } \\
\text { ated with a judgment point, was not coded }\end{array}$ \\
\hline Expectancies & $\begin{array}{l}\text { Participants' mental representations of the relation- } \\
\text { ships between cues and judgments. Includes both } \\
\text { deliberative and elicited implicit reasoning }\end{array}$ \\
\hline Goals & $\begin{array}{l}\text { The participants indicated aim at each point of } \\
\text { judgment, or explanation for an action }\end{array}$ \\
\hline Action & $\begin{array}{l}\text { Actions taken, ie, details of history taking, examina- } \\
\text { tion, referral, etc }\end{array}$ \\
\hline
\end{tabular}


and believed that colleagues would have assessed them differently. The intensity of gut feelings, despite the underlying uncertainty, is reflected in their decisions, which often went against their colleagues' advice:

So I rang the [hospital doctor] on-call and said I'd like him to see this patient, very urgently. And he went through with me, the history, the clinical findings, and he said, "I don't agree with you, I don't think this man's got anything serious." So I just said, "If you're not willing to see him, I'm going to send him to A \& E [Accident and Emergency]," which in fact we had to do (physician 5 talking about a 28 -year-old male patient with flulike symptoms. Final diagnosis: meningococcal septicemia).

\section{Recognitions}

In recognition cases, a diagnosis is formulated quickly and with little information. Although recognition cases appear similar to first impressions, physicians were aware of conflicting information and/or the absence of key symptoms and signs. Some thought that colleagues would consider their judgment unwarranted. They nevertheless remained confident in their intuition:

I was thinking, "Probably, I should investigate his chest pain." But slightly reluctantly, I suppose, because I thought, I only saw anxiety. I still thought there was nothing wrong (physician 15 talking about a highly-anxious, 46-year-old male patient, reporting chest pain and breathlessness. Final diagnosis: heart disease).

\section{Insights}

In this type of case, no pattern of cues is recognizable initially, and no satisfactory interpretation is formed, although several diagnoses are considered, and the physician engages in extensive information gathering. A clear interpretation is suddenly and rapidly formed that integrates and explains all the symptoms and signs, sometimes as the direct result of a single piece of information from long-term memory suddenly coming into the physician's awareness. Physicians were surprised and were not aware of how the insight had happened:

I wasn't really focused on that.... We were just talking and it suddenly flashed in my head. Something I'd read somewhere...something about lupus connected with celiac disease. And so I got really excited.... I couldn't even remember where I'd read it (physician 9 talking about a 50 -year-old female patient with existing cutaneous lupus erythematosus and irritable bowel syndrome, complaining of exacerbated symptoms).

\section{Experienced Conflict}

In all cases and process types, physicians talked about experiencing conflict between their intuition and another interpretation that they considered more ratio- nal. This conflict included those reporting insights who, despite feeling they had arrived at an explanation, still believed that colleagues would not be convinced (Supplemental Appendix, physician 3). Three sources of conflict were apparent: (1) the diagnosis arrived at intuitively was considered highly unlikely, either because it seemed implausible, eg, colorectal cancer in a young patient, or it was a rare disease in the general population, eg, pancreatic cancer $;$ (2) the cue that seemed to lead to the intuition was out of the ordinary and not evidence based, eg, spontaneous painless rectal bleeding, lymph nodes that felt too large or too hard or not in the right place-the physicians did not know what the cues meant but knew that they did not fit with their initial hypothesis or a previous diagnosis; (3) cue patterns that seemed to lead to intuitions were complex and difficult to verbalize. For example, a patient consulted with flulike symptoms that appeared the evening before. He thought he had influenza, felt very weak and unwell, and had a headache; however, he had no respiratory or gastrointestinal symptoms consistent with influenza. Although his symptoms individually would not cause alarm, the pattern of symptoms present and absent caused a feeling of unease and alerted the physician to examine more closely_-including an eye examination for papilledema-and eventually to diagnose an early case of meningococcal septicemia.

Physicians reported only 7 cases for which their intuitions turned out to be incorrect. All process types contained examples of incorrect intuition. Cases of gut feelings were reported more frequently by experienced (more than 10 years in practice) than by less experienced physicians and by female more often than by male physicians.

\section{DISCUSSION}

The medical literature discusses clinical intuition as diagnosis based on first impressions. ${ }^{10,12,14,31}$ Although this process is undoubtedly present and influential in physicians' decision making, our results suggest that it may play only a part in what most family physicians understand by the term intuition. We identified 3 types of decision processes that need to be investigated further. Different theoretical perspectives seem best suited to the different types of intuitive processes that we propose.

First, gut feelings appear to be similar to the slowing down observed by surgeons, who realize that they need to be more deliberative at a certain moment during an operation but cannot always explain why. ${ }^{32}$ One explanation for this type of effect is that new, conflicting information is harder to process and integrate into the coherent representation which routine 
decision making requires. ${ }^{33,34}$ As a result, individuals experience a meta-cognitive feeling of unease or uncertainty, which acts as a signal that more deliberation is needed to integrate all the information. ${ }^{23,35}$ Physicians who reported experiencing a gut feeling that all was not well maintained a cautious approach, but subsequent deliberation did not resolve the feeling of unease. This feeling of unease could be due to implicit knowledge being active at the time. The learning perspective on intuition focuses on the role of implicit knowledge, learned through experience, which is not consciously available and thus forms the basis of intuition. ${ }^{36}$ The importance of experience is reflected in our finding that more experienced family physicians reported relatively more gut feeling cases than those less experienced. This learning perspective also points out that the validity of intuition relies not only on the length of clinical experience but also, perhaps more importantly, on the quality and frequency of feedback during learning. Poor feedback leads to poor intuition, whereas good feedback leads to good intuition. ${ }^{37}$ Finally, this perspective emphasizes the role of emotion: learning is strongest when associated

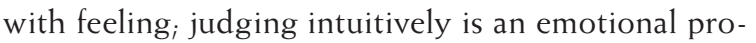
cess whereby the judgment is felt. ${ }^{5,38}$

Second, recognition seems to be encompassed by the heuristics approach, where quick pattern recognition based on salient features and simple rules forms the basis for an initial judgment. 4,39,40 Often, this process goes unnoticed, but in situations where there is a conflict between the physician's judgment and the available information, it might be described by the physician as an intuition. In addition, evidence suggests that decision makers are biased to their initial, automatic hypothesis. ${ }^{41}$ This bias is dependent on the perceived strength of conflicting information. Information that is not salient in the environment, such as base rates, is likely to exert less influence on judgments than highly salient information, such as the presence of a typical or diagnostic feature.

Third, insight-type intuitions may be best explained by the literature on incubation, which suggests possible mechanisms for insightful problem solving after failures to solve a problem with analytical thinking. These mechanisms include forgetting irrelevant information and changing the mental representation of the problem. ${ }^{42}$ The incubation literature assumes the "Aha!" experience is due to the passing of time between failed attempts to solve a problem analytically, but new experimental paradigms in psychology suggest that engaging attention away from the problem at hand, even for a few minutes, may be sufficient for its successful solution. ${ }^{24}$ The mechanisms for this phenomenon are still a matter of debate. ${ }^{43-45}$
Our study has a number of strengths. Rather than asking physicians' opinions about the value and use of intuition in clinical practice, we examined their decision processes in specific patient cases. Physicians themselves identified these as cases where their judgment was based on intuition rather than analysis, which ensures that our findings have external validity for physicians and are not researchers' conceptualizations that we imposed unchecked on clinical practice. Furthermore, the use of the Critical Decision Method guided the elicitation of detailed and internally consistent case descriptions that included both explicit and implicit elements of the decision process. ${ }^{29}$ As a result, we were able to search for similarities in the decision process of different cases: the timing of the intuition (earlier vs later in the process), the amount of information elicited before the intuition (minimal vs substantial), the existence of alternative explanations (single vs multiple), and the perceived conflict between what the physician believed was the right judgment and what a more rational approach or other colleagues would suggest.

We also acknowledge the limitations to our study. First, the first author conducted both data collection and data analysis, which had the potential to influence the way that the interview was conducted and the data interpreted. To guard against undue influence, the interview schedule was designed by both authors on the basis of a standard instrument (Critical Decision Method). The second author categorized all transcripts independently into the emerging scheme, and agreement was subsequently reached. This reduced the likelihood that one author's assumptions about the cognitive processes present at the time of intuition influenced case classification. Furthermore, the interviewer was not medically trained and was thus less likely to make assumptions about the physicians' reasoning or prompt their responses. Finally, the study was exploratory, without any previous hypotheses about the types of cognitive processes to be expected and without prior interest in specific outcomes.

The very method of the study, case recall, has limitations. Physicians had reflected upon the cases, attempting to understand the experienced intuition, as a result, they may have rationalized it. Accordingly, some cases that physicians attribute to intuition, upon subsequent reflection and description during the interview, may end up appearing as the product of rational and purposeful thought. Furthermore, individuals' awareness of and ability to report their cognitive processes accurately has been called into question. ${ }^{46,47}$ The Critical Decision Method was chosen because it specifically addresses the potential for bias in retrospective reports. Hoffman and colleagues report that $82 \%$ of key elements of experts' accounts corre- 
sponded with an earlier account and that independent coders extract the same information from Critical Decision Method protocols. ${ }^{29}$

Clinical intuition encompasses a variety of cognitive processes that go beyond mere first impressions. It can occur at any point during the clinical encounter and with differing amounts of information. In the cases discussed, physicians experienced conflict between their intuition and a decision that they perceived to be more rational, or between their intuition and their expectations about what other physicians would do. The outcomes of clinical intuition can be negative but they can also be positive, as the plethora of examples in this study suggests. Until we further specify the circumstances under which intuitive processes produce accurate judgments, there cannot be a simple, catchall directive to physicians to avoid intuition.

To read or post commentaries in response to this article, see it online at http://www.annfammed.org/content/11/1/60.

Key words: decision making; judgment; diagnosis; intuition; family practice; Critical Decision Method

Submitted December 12, 2011; submitted, revised, May 11, 2012; accepted May 24, 2012.

Funding support: This research was supported by the National Institute for Health Research (NIHR) Biomedical Research Centre at Guy's and St Thomas' NHS Foundation Trust and King's College London. Amanda Woolley was supported by a departmental PhD studentship. Olga Kostopoulou was supported by a Guy's \& St Thomas' Charity senior lectureship (associate professorship).

Disclaimer: The views expressed are those of the authors and not necessarily those of the NHS, the NIHR or the Department of Health.

Previous presentations: Portions of this article have been presented at the meeting of the European Group of Process Tracing studies, Greifswald, Germany, May 5-7, 2011 (EGPROC 2011); annual conference of the Society for Academic Primary Care (SAPC), Bristol, UK, July 6-8, 2011; and the Subjective Probability, Utility and Decision Making meeting, Kingston UK, August 21-25, 2011 (SPUDM 2011).

Acknowledgments: The authors wish to thank the academic family physicians that helped with the understanding of the clinical content of the cases: Shyamalee Samaranayaka, Brendan Delaney, Thomas Round, and Ellen Wright. We also gratefully acknowledge the contribution of the participating family physicians.

\section{References}

1. Greenhalgh T. Intuition and evidence-uneasy bedfellows? $\mathrm{Br} J \mathrm{Gen}$ Pract. 2002;52(478):395-400.

2. Barraclough K. Medical intuition. BMJ. 2006;332:497.2.

3. Spence D. May the force be with you. BMJ. 2007;335:1158.1.

4. Gigerenzer G, Todd PM, The ABC Research Group. Simple Heuristics That Make us Smart. New York, NY: Oxford University Press; 1999.

5. Betsch T, Plessner H, Schwieren C, Gutig R. I like it but I don't know why: a value account approach to implicit attitude formation. Pers Soc Psychol Bull. 2001;27(2):242-253.
6. Klein GA. Sources of Power: How People Make Decisions. Cambridge, MA: MIT Press; 1999.

7. Hammond KR, Hamm RM, Grassia J, Pearson T. Direct comparison of the efficacy of intuitive and analytical cognition in expert judgment. IEEE Trans Syst Man Cybern. 1987;17(5):753-770.

8. Dijksterhuis A, Bos MW, Nordgren LF, van Baaren RB. On making the right choice: the deliberation-without-attention effect. Science. 2006;311(5763):1005-1007.

9. Wilson TD, Schooler JW. Thinking too much: introspection can reduce the quality of preferences and decisions. J Pers Soc Psychol. 1991;60(2):181-192.

10. Hall $\mathrm{KH}$. Reviewing intuitive decision-making and uncertainty: the implications for medical education. Med Educ. 2002;36(3):216-224.

11. Croskerry P. Critical thinking and decisionmaking: avoiding the perils of thin-slicing. Ann Emerg Med. 2006;48(6):720-722.

12. Croskerry P. The importance of cognitive errors in diagnosis and strategies to minimize them. Acad Med. 2003;78(8):775-780.

13. Klein JG. Five pitfalls in decisions about diagnosis and prescribing. BMJ. 2005;330(7494):781-783.

14. Mamede S, Schmidt HG, Penaforte JC. Effects of reflective practice on the accuracy of medical diagnoses. Med Educ. 2008;42(5): $468-475$.

15. Kulatunga-Moruzi C, Brooks LR, Norman GR. Coordination of analytic and similarity-based processing strategies and expertise in dermatological diagnosis. Teach Learn Med. 2001;13(2):110-116.

16. Eva KW, Hatala RM, Leblanc VR, Brooks LR. Teaching from the clinical reasoning literature: combined reasoning strategies help novice diagnosticians overcome misleading information. Med Educ. 2007;41(12):1152-1158.

17. Stolper $E$, van Bokhoven $M$, Houben $P$, et al. The diagnostic role of gut feelings in general practice. A focus group study of the concept and its determinants. BMC Fam Pract. 2009;10:17.

18. Shah AK, Oppenheimer DM. Heuristics made easy: an effort-reduction framework. Psychol Bull. 2008;134(2):207-222.

19. Gilovich T, Griffin D, Kahneman D. Heuristics and Biases: The Psychology of Intuitive Judgment. New York, NY: Cambridge University Press; 2002.

20. Goldstein DG, Gigerenzer G. Models of ecological rationality: the recognition heuristic. Psychol Rev. 2002;109(1):75-90.

21. Kahneman D. A perspective on judgment and choice: mapping bounded rationality. Am Psychol. 2003;58(9):697-720.

22. De Neys W, Glumicic T. Conflict monitoring in dual process theories of thinking. Cognition. 2008;106(3):1248-1299.

23. Thompson VA. Dual process theories: a metacognitive perspective. In: Evans J, Frankish K, eds. In Two Minds: Dual Processes and Beyond. Oxford: Oxford University Press; 2009.

24. Dijksterhuis A, Nordgren LF. A theory of unconscious thought. Perspect Psychol Sci. 2006;1(2):95-109.

25. Reber AS. Implicit Learning And Tacit Knowledge: An Essay on the Cognitive Unconscious. New York, NY; Oxford: Oxford University Press; 1993.

26. Frederick S. Cognitive reflection and decision making. J Econ Perspect. 2005;19(4):25-42.

27. Simon HA, Chase WG. Skill in chess. Am Sci. 1973;61(4):394-403.

28. Ericsson KA. Deliberate practice and the acquisition and maintenance of expert performance in medicine and related domains. Acad Med. 2004;79(10)(Suppl):S70-S81.

29. Hoffman RR, Crandall B, Shadbolt N. Use of the Critical Decision Method to elicit expert knowledge: a case study in the methodology of cognitive task analysis. Hum Factors. 1998;40(2):254-277.

30. Klein GA, Calderwood R, Macgregor D. Critical Decision Method for eliciting knowledge. IEEE Trans Syst Man Cybern. 1989;19(3):462-472. 
31. Norman G, Young M, Brooks L. Non-analytical models of clinical reasoning: the role of experience. Med Educ. 2007;41(12):1140-1145.

32. Moulton CA, Regehr G, Lingard L, Merritt C, MacRae H. Slowing down to stay out of trouble in the operating room: remaining attentive in automaticity. Acad Med. 2010;85(10):1571-1577.

33. Betsch T, Glockner A. Intuition in judgment and decision making: Extensive thinking without effort. Psychol Inq. 2010;21(4):279-294.

34. Alter AL, Oppenheimer DM. Uniting the tribes of fluency to form a metacognitive nation. Pers Soc Psychol Rev. 2009;13(3):219-235.

35. Thompson VA, Prowse Turner JA, Pennycook G. Intuition, reason, and metacognition. Cogn Psychol. 2011;63(3):107-140.

36. Betsch T. The nature of intuition and its neglect in research on judgment and decision making. In: Plessner $\mathrm{H}$, Betsch $\mathrm{C}$, Betsch $\mathrm{T}$, eds. Intuition in Judgment and Decision Making. New York, NY: Psychology Press; 2008.

37. Hogarth RM. Educating Intuition. Chicago, IL: University of Chicago Press; 2001.

38. Epstein S. Integration of the cognitive and the psychodynamic unconscious. Am Psychol. 1994;49(8):709-724.

39. Kahneman D, Frederick S. Representativeness revisited: attribute substitution in intuitive judgment. In: Gilovich T, Griffin D, Kahneman D, eds. Heuristics and Biases: The Psychology of Intuitive Judgment. New York, NY: Cambridge University Press; 2002:49-81.
40. Schmidt HG, Rikers RM. How expertise develops in medicine: knowledge encapsulation and illness script formation. Med Educ. 2007;41(12):1133-1139.

41. Simmons JP, Nelson LD. Intuitive confidence: choosing between intuitive and nonintuitive alternatives. J Exp Psychol Gen. 2006;135 (3):409-428.

42. Sio UN, Ormerod TC. Does incubation enhance problem solving? A meta-analytic review. Psychol Bull. 2009;135(1):94-120.

43. Gonzalez-Vallejo C, Lassiter GD, Bellezza FS, Lindberg MJ. "Save angels perhaps": a critical examination of unconscious thought theory and the deliberation-without attention effect. Rev Gen Psychol. 2008;12(3):282-296.

44. Calvillo DP, Penaloza A. Are complex decisions better left to the unconscious? Further failed replications of the deliberation-withoutattention effect. Judgm Decis Mak. 2009;4(6):509-517.

45. Waroquier L, Marchiori D, Klein O, Cleeremans A. Methodological pitfalls of the Unconscious Thought paradigm. Judgm Decis Mak. 2009;4(7):601-610.

46. Nisbett RE, Wilson TD. Telling more than we can know-verbal reports on mental processes. Psychol Rev. 1977;84(3):231-259.

47. Ericsson KA, Simon HA. Protocol Analysis: Verbal Reports as Data. Cambridge, MA: MIT Press; 1993. 